\title{
STRAIN LOCALIZATION PHENOMENA IN GENERALIZED SINGLE CRYSTAL PLASTICITY
}

\author{
Samuel Forest \\ Ecole des Mines de Paris / CNRS, Centre des Materiaux UMR 7366, BP 87, 91003 Evry, France
}

\begin{abstract}
An account of recent progress in strain localization phenomena in generalized single crystal plasticity is given.
\end{abstract}

\section{Introduction}

The validity of a constitutive model in materials mechanics is often assessed in regard of its ability to satisfactorily describe the homogeneous deformation of the material under various loading conditions. In crystal plasticity much attention has been focused on the anisotropic tensile, compression and shear behaviour of single crystals. In each case maintaining a zone of homogeneous deformation in a specimen during testing turns out to be a difficult experimental task. The objective of the present work is to show that the question of the pertinence of such mechanical modelling can only be settled if the model response is considered under conditions for which deformation becomes non-homogeneous. For that purpose, structural calculations are needed in order to work out the associated strain and stress fields. The confrontation with experiments is then all the more difficult that local strain measurements are necessary [1]. Nonhomogeneous deformation may arise as a result of particular loading conditions (like torsion investigated in [2] and [3]) or, more drastically, of a bifurcation from an initially homogeneous state. A bifurcation analysis of the retained constitutive equations provides valuable information for the critical assessment of the model. In the following, some successes of classical crystal plasticity in predicting observed localization modes are presented and some shortcomings are shown that can be solved by resorting to the mechanics of generalized continua.

\section{Classical treatment of strain localization phenomena in single crystals}

\subsection{Local softening mechanisms}

The evidence of instabilities is overwhelming in the field of plastic deformation of metals. Applied stresses induce self-organization of dislocation populations that may lead to localization of deformation. An understanstanding of the collective behaviour of dislocations can be reached through a stability analysis of various physical mechanisms involved at the microscale. Walgraef and Aifantis [4] developed these technics to describe pattern formation in single crystals. As for them, Estrin and Kubin [5] have investigated averaged properties of dislocations over a local volume in order to compute a local strain hardening. They found that these mechanical properties 
differ from the bulk properties obtained by measuring the overall strain hardening in a macroscopic specimen. This difference is especially pronounced at small strains and can be attributed to the inherently non-uniform character of plastic slip. It turns out that, at the very beginning of plastic flow, local strain softening occurs, which means that plastic deformation will necessary start in a non-uniform manner.

\subsection{Bifurcation analysis}

Since Asaro and Rice [6], the occurrence of shear bands in single crystals has been described by a bifurcation analysis of the set of constitutive and balance equations. They performed such a bifurcation analysis at large strains for elastoplastic single crystals undegoring single slip. They found two possible localization modes : slip and kink bands. For slip bands the localization plane coincides with the glide plane. For kink bands the localization plane is normal to the slip direction of the considered system. These two localization modes become possible for a vanishing critical hardening modulus if only small strains are considered. The case of 2,4,6 or 8 simultaneously activated slip systems in f.c.c. single crystals in tension has been investigated in [7].

\subsection{Slip band propagation}

We introduce a short softening period followed by subsequent hardening in the single crystal behaviour (figure la). The softening stage promotes strain localization whereas subsequent hardening leads to shear band propagation. These heterogeneous deformation modes have been simulated using the finite element method for a single crystal plate in tension undergoing single slip. The abrupt fall on the load-displacement curve (figure $\mathrm{lb}$ ) indicates that localization occurs. The load then remains constant. At the same time, slip bands spread over the whole specimen (figure 2 and 3 ). When the entire specimen is covered with slip bands, the load increases again. The load-displacement curve of figure $\mathrm{lb}$ can be compared with experimental results obtained at BAM (Berlin, Germany) for single crystal superalloy $\mathrm{SCl} 6$ at room temperature [8]. In this latter case, the propagation of slip bands along the specimen has been seen and filmed. In general, tensile curves displaying a short plateau often are an indicator of strain localization and propagation followed by homogeneous hardening. Such curves should not be interpreted as giving the intrinsic behaviour of the material but rather as a structural effect.

\subsection{Symmetry-breaking modes}

The orientation of shear bands in single crystals oriented for symmetric multiple slip has been found to be non-crystallographic in [7]. However the stability of symmetric multislip configurations strongly depends on the ratio of self- and latent hardening moduli, and symmetrybreaking situations have been simulated in [9] : slip and kink bands for each slip system indefferently form instead of shear bands involving both slip systems. 

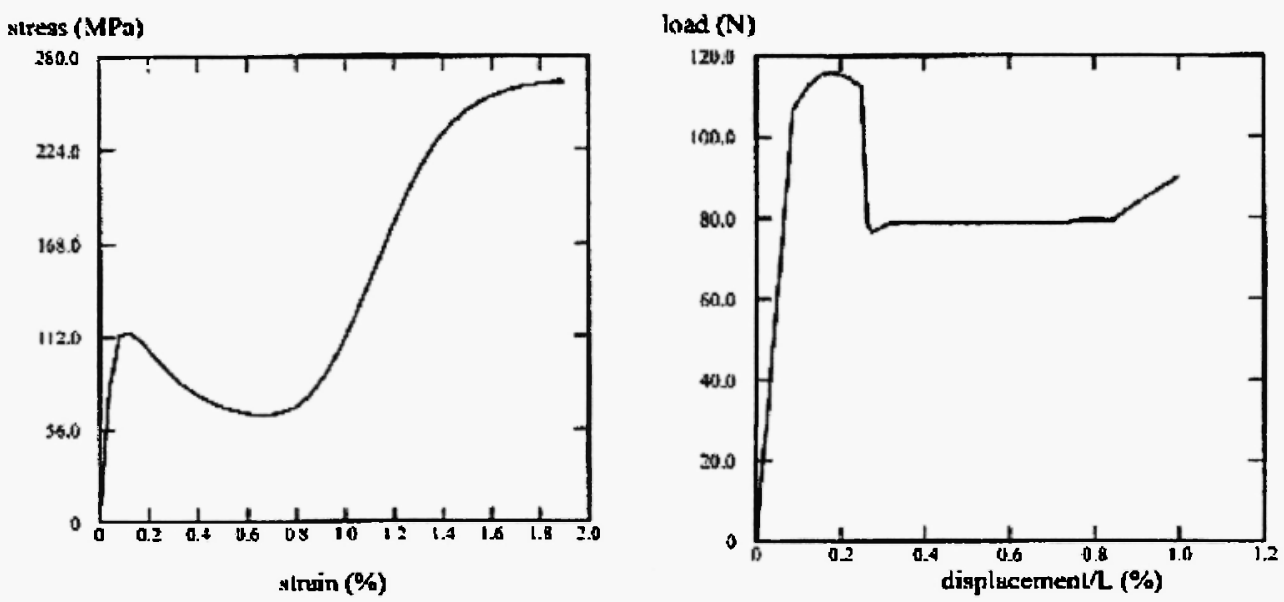

Figure 1 : Strain-softening behaviour of a single crystal in tension (left) and global response of a single crystal plate in tension (right).

\section{Cosserat single crystal plasticity}

\subsection{Motivations}

The classical theory of crystal plasticity with internal variables [10] has proved to be an efficient and accurate framework to describe the material response of single crystals undergoing homogeneous deformation and even in the situations of localized deformation investigated earlier. However, the internal variables or hardening variables are related to the densities of dislocations $\rho$ which represent only a crude description of dislocation population inside a volume element. Kröner suggested to consider correlation functions of the dislocation distribution [11]. The first correlation function is the so-called dislocation density tensor introduced by Nye [12] and represents the main variable of the continuum theory of dislocations. It does not play any significant role in the homogeneous hardening of crystals but may become dominant in the case of strongly non-homogeneous deformation. Note that $\rho$ is nothing but an invariant of the second order correlation function. As a result $\rho$ and $\alpha$ represent two independent moments of the same distribution. A more refined theory of crystal plasticity should introduce both variables.

The dislocation density is related to the rotational part of plastic deformation so that a strain gradient formulation is adequate to introduce $\alpha$ as in [13]. However, $\alpha$ is also linked to lattice curvature $\kappa$ via Nye's relation. This suggests that a single crystal can be regarded as a Cosserat medium.

\subsection{Constitutive framework}

A full finite strain and curvature constitutive framework for Cosserat single crystal elastoviscoplasticity has been reported in [14]. The main features are the following. 

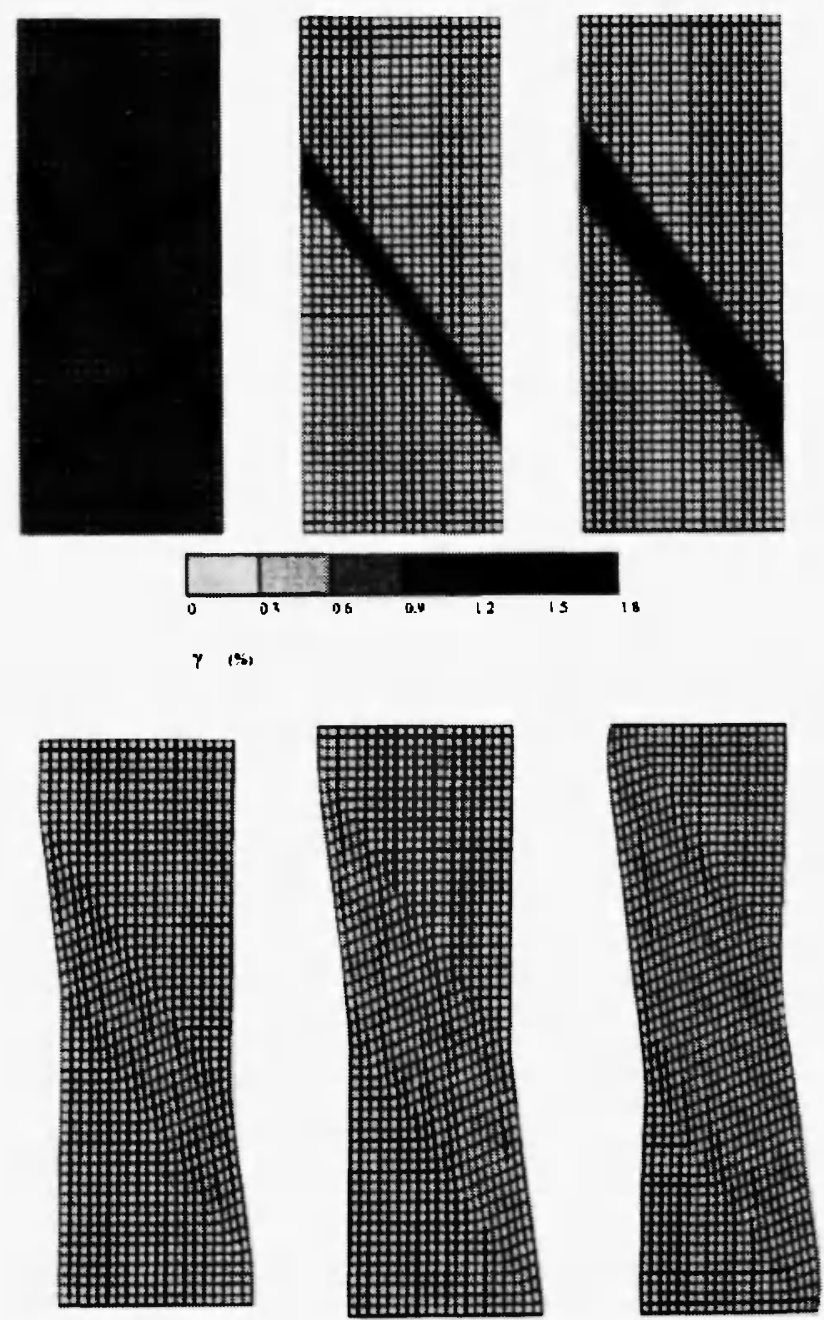

Figure 2 and 3 : Slip band formation and propagation in a single crystal plate oriented for single slip in tension : slip amount (above, two defects have been introduced), deformed state (below).

Displacements of material points and the rotation of the underlying lattice basis in its released state are treated as independent degrees of freedom.

The usual multiplicative decomposition of the deformation gradient into its elastic and plastic parts is introduced whereas total lattice curvature is split into elastic and plastic parts in a quasi-additive manner. Plastic deformation is the result of slip processes according to slip systems. Similarily plastic lattice curvature and torsion can develop only according to special crystallographic directions that can be idealized by directions associated with pure edge and screw dislocations.

The activation of slip systems is governed by Schmid law. In the same way, the resolved couple stresses are responsible for the development of plastic lattice curvature and torsion and a generalized Schmid law is shown to exist. Additional hardening due to plastic curvature is finally introduced. A viscoplastic framework is chosen to avoid any indeterminacy in the activation of slip systems. 


\section{Application to kink banding in single crystals}

\subsection{Bifurcation analysis}

A rather puzzling aspect of classical crystal plasticity that arises from the localization analysis of section 1 is that it predicts that slip and kink bands can appear simultaneously for the same critical hardening modulus. But slip and kink bands are very different localization modes from the physical point of view. Kink banding is associated with strong lattice curvature whereas slip bands do not affect crystal orientation. Kink bands are seldom observed at the beginning of the plastic flow although they may develop at larger strains. In contrast slip bands are the main plasticity mechanisms. The fact that classical crystal plasticity does not really distinguish the onsets of slip and kink banding should regarded as a shortcoming.

A bifurcation analysis for Cosserat single crystals undergoing single slip shows that these two different modes can be separated. Slip bands can still occur for a vanishing hardening modulus but additional hardening associated with lattice curvature then postpones kink band formation as proved in [9].

\subsection{FE simulations of Cosserat crystals}

The Cosserat model has been implemented in a finite element code and the localization calculations of the first section have been reconsidered. Note that such a computation leads to twice more degrees of freedom and three times more internal variables than the corresponding classical one. For a single crystal plate oriented for single slip, it appears that, due to additional hardening associated with plastic lattice curvature, kink bands are precluded and slip band bundles form instead [9].

A major issue of Cosserat modelling of crystals is the determination of the additional parameters involved. In the isotropic case, at least four additional parameters are necessary. Two of them concern Cosserat elasticity. The parameter $\mu \mathrm{c}$ [9] governs the intensity of the skewsymmetric part of the force-stress tensor and a bending stiffness must be attributed to the crystal. A characteristic length enters the formulation of plastic curvature flow and is thougth to be the specific size of the retained volume element of Cosserat mechanics (it has a finite size !). Below this size dislocation distributions are not distinguished. It depends on the final structural calculation one aims at. An additional hardening modulus linking curvature to slip can then be introduced. We claim that this parameter could be identified, using simulations of dislocation dynamics under non-homogeneous deformation conditions.

\subsection{Should slip bands be regularized ?}

It turns out that slip bands are left unaffected by Cosserat plasticity since they are not necessarily associated with lattice curvature. The simulation of shear bands using the finite element method is known to lead to a spurious mesh-dependence [7]. Cosserat crystal plasticity has been shown to regularize kink bands that will then have a finite width. This is not the case for slip bands. It must be noted that the strain gradient theory presented in [13] does not affect slip bands for the same reasons as for our theory. 


\section{References}

[1] Ziebs J., Bressers J., Frenz H., Hayhurst D.R., Klingelhöffer H., Forest S., Local strain and temperature measurements in non-uniform fileds, Woodhead Publishing Limited, 1996.

[2] Nouailhas D., Cailletaud G., Int. J. Plasticity, 11 (4), pp.451-470, 1995.

[3] Forest S., Olschewski J., Ziebs J., Kühn H.J., Meersmann J., Frenz H., Fatigue 6, Lütjering G. and Novak H., Pergamon, pp. 1087-1092, 1996.

[4] Walgraef D., Aifantis E.C., Res Mechanica, 23, pp. 161-195, 1988.

[5] Estrin Y., Kubin L., Acta Metall., 34 (12), pp. 2455-2464, 1988.

[6] Asaro R.J., Rice J.R., J. Mech. Phys. Solids, 25, pp. 309-338, 1977.

[7] Forest S., Cailletaud G., Eur. J. Mech. A/Solids, 14(5), pp. 747-771, 1995.

[8] Brite Euram project BE 5216, coordinated by J. Olschewski, BAM, Berlin, 1995.

[9] Forest S., Acta Mat., 46(9), pp. 3265-3281, 1998.

[10] Mandel J., Int. J. Solids Structures, 9, pp. 725-740, 1973.

[11] Kröner E., in Inelastic Behaviour of solids, Kanninen M.F., Adler W.F., Rosenfield A.R. ed. , McGraw-Hill, pp. 137-147, 1969.

[12] Nye J.F., Acta Metall., 1, pp. 153-162, 1953

[13] Shu J.Y., Fleck N.A., King W.E., MRS Fall Meeting Proc. Symposium W, 1996.

[14] Forest S., Cailletaud G., Sievert R., Arch. Mech., 49(4), 705, 1997. 